QUARTERLY OF APPLIED MATHEMATICS

VOLUME LXVII, NUMBER 3

SEPTEMBER 2009, PAGES 559-578

S 0033-569X(09)01153-9

Article electronically published on May 6, 2009

\title{
TRAVELING WAVES \\ OF TWO-COMPONENT REACTION-DIFFUSION SYSTEMS ARISING FROM HIGHER ORDER AUTOCATALYTIC MODELS
}

\author{
BY
}

JONG-SHENQ GUO (Department of Mathematics, National Taiwan Normal University, 88, Section 4, Ting Chou Road, Taipei 116, Taiwan)

AND

JE-CHIANG TSAI (Department of Mathematics, National Chung Cheng University, 168, University Road, Min-Hsiung, Chia-Yi 621, Taiwan)

Abstract. We study the existence and uniqueness of traveling wave solutions for a class of two-component reaction-diffusion systems with one species being immobile. Such a system has a variety of applications in epidemiology, bio-reactor models, and isothermal autocatalytic chemical reaction systems. Our result not only generalizes earlier results of Ai and Huang (Proceedings of the Royal Society of Edinburgh 2005; 135A:663-675), but also establishes the existence and uniqueness of traveling wave solutions to the reactiondiffusion system for an isothermal autocatalytic chemical reaction of any order in which the autocatalyst is assumed to decay to the inert product at a rate of the same order.

1. Introduction. In this article, we are concerned with the following reactiondiffusion system:

$$
u_{t}=d_{1} u_{x x}-f(u) v^{m}, \quad v_{t}=d_{2} v_{x x}+[f(u)-K] v^{m} .
$$

Here $d_{1} \geq 0, d_{2} \geq 0, K>0$ and $m \geq 1$ are constants, and $f$ is a differentiable function defined on $(0, \infty)$. The system (1.1) has been proposed as a model for several physical and biological systems, which we shall briefly discuss below.

First, if we choose $f(u)=\beta u$ and $m=1$, then the system (1.1) is a diffusive epidemic model, which describes the interaction between the susceptible individual $u$ and the

Received March 13, 2008.

2000 Mathematics Subject Classification. Primary 34A34, 34A12; Secondary 35K57.

Key words and phrases. Traveling waves, reaction-diffusion systems, centre manifold.

The first author was supported in part by the National Science Council of the Republic of China under the contracts NSC 96-2119-M-003-001.

The second author is the corresponding author and was partially supported by the National Science Council of the Republic of China under the contracts NSC 96-2115-M-194-003-MY3.

E-mail address: jsguo@math.ntnu.edu.tw

E-mail address: tsaijc@math.ccu.edu.tw 
infected individual $v$. In this case, the reaction term $\beta u v$ is of the well-known KermackMcKendric type [17, 6]. Moreover, the positive constants $\beta$ and $K$ are the contact rate and the removal rate, respectively.

Secondly, if $m=1$ and $f(u)$ takes the form $m_{1} u /\left(m_{2}+u\right)$ with positive constants $m_{1}$ and $m_{2}$, then the system (1.1) has been introduced in [7. to study the effects of motility on the ability of a bacterial population to survive in a flow reactor and/or to be a good competitor for a limiting nutrient in a mixed culture if we set the flow velocity to be zero. In this situation, $u$ stands for the concentration of the nutrient, $v$ is the density of the bacterial population, and $f(u)$ describes the nutrient uptake and growth rate of the bacterial population at the nutrient concentration $u$. The parameter $K>0$ is the cell death rate. We remark that Kennedy and Aris [18 have also considered a similar bio-reactor model.

The parameter $m$ is chosen to be 1 in the previous two applications. But, the parameter $m$ can be different from 1 in the context of higher order autocatalytic reactions. Specifically, we follow Merkin and Needham 21, 20, 19] to consider the general $m$ th order isothermal autocatalytic chemical reaction

$$
A+m B \rightarrow(m+1) B \quad \text { with rate } k_{1} u v^{m}
$$

with the autocatalyst $B$ assumed to decay to the inert product $C$ at a rate of order $m$,

$$
B \rightarrow C \text { with rate } k_{2} v^{m},
$$

where $u$ and $v$ are the concentration of the reactant $A$ and the autocatalyst $B$, respectively, and the $k_{i}$ are the rate constants. Then by applying the law of mass action, the above reaction schemes lead to the system (1.1) with $f(u)=k_{1} u$ and $K=k_{2}$. Unlike the previous examples, here $m$ can be any positive integer. Such autocatalytic reaction schemes have been used in many models of realistic chemical systems. For example, it has been shown by Voronkov and Semenov [26] that the cubic autocatalytic reaction (the reaction (1.2) with $m=2$ ) can be used to model the almost-isothermal flames in the carbon-sulphide-oxygen reaction. The cubic autocatalytic reaction also works well in the iodate-arsenous acid system proposed by Saul and Showalter 24]. Furthermore, Aris, Gray and Scott [3] have discussed that a series of bimolecular steps can lead to the cubic autocatalytic reaction. On the other hand, if we ignore the effect of the decay to the inert product, then the constant $K$ becomes zero in (1.1). For this case, we refer the reader to the works of Billingham and Needham [4, 5, Qi [23, Ai and Huang [2] and Chen and Qi 9. However, as Gray [11] suggested, the decay step (1.3) plays an important role in these autocatalytic chemical reactions. Therefore, we shall take this step into our consideration in this paper.

Wave phenomena have been observed in a variety of chemical and biomedical sciences (see 22]). For example, experimental observations 28] indicate that traveling waves can be generated in the chemical system based on the cubic autocatalytic chemical reaction followed by the quadratic decay. Specifically, suppose that initially we have the reactant $A$ at uniform concentration, and introduce a quantity of the autocatalyst, $B$, locally into this system. Then $A$ and $B$ will react in this initial zone, which results in the concentration gradients and then leads to the generation of two waves propagating from 
this initial zone for the reactant $A$ (and the autocatalyst $B$, respectively). The wave for the reactant is of front type and the one for the autocatalyst is of pulse type due to the decay. By focusing on the right-half part, this leads to the study of the problem of traveling waves. Mathematically, it has also been known [27] that traveling waves of the reaction-diffusion systems play a key role in understanding the dynamical behavior of solutions of the system under study. These two reasons and the rich applications of the system (1.1) necessitate a detailed study of traveling wave solutions of (1.1).

We first define what we mean by a traveling wave solution of the system (1.1). A couple of nonnegative functions $(U, V) \in C^{2}(\mathbb{R}) \times C^{1}(\mathbb{R})$ is said to be a traveling wave solution of (1.1) with wave speed $c \in \mathbb{R}$ if $(u(x, t), v(x, t))=(U(z), V(z)), z:=x-c t$, is a solution of (1.1) and satisfies the boundary conditions $\lim _{z \rightarrow-\infty}(U, V)=(a, 0)$ and $\lim _{z \rightarrow+\infty}(U, V)=(b, 0)$ for some $a \in(0, \gamma)$ and $b \in(\gamma, \infty)$, where $\gamma$ is a positive constant satisfying $f(\gamma)=K$. That is, $(U, V)$ satisfies the ordinary differential system

$$
d_{1} U^{\prime \prime}+c U^{\prime}-f(U) V^{m}=0, \quad d_{2} V^{\prime \prime}+c V^{\prime}+[f(U)-K] V^{m}=0
$$

subject to the boundary conditions

$$
\lim _{z \rightarrow-\infty}\left(U, U^{\prime}, V, V^{\prime}\right)=(a, 0,0,0), \quad \lim _{z \rightarrow+\infty}\left(U, U^{\prime}, V, V^{\prime}\right)=(b, 0,0,0),
$$

where the prime denotes $d / d z$.

The existence of traveling wave solutions of (1.1) has been investigated by several authors. When $d_{1}=0$ and $m=1$, the problem (1.4)-(1.5) can be reduced to a system of two first order ordinary differential equations, and Källén [16] and Kennedy [18 have used the phase-plane analysis to solve the existence and uniqueness of solutions of the problem (1.4) - (1.5) for the particular choice $f(u)=u$ and $f(u)=m_{1} u^{m_{2}} /\left(m_{3}+u^{m_{2}}\right)$ with some positive constants $m_{1}, m_{2}$, and $m_{3}$, respectively. For sufficiently small $d_{1}>0$ and $m=1$, Smith and Zhao 25] have employed the so-called geometric singular perturbation theory to treat the case when $f(u)=m_{1} u /\left(m_{2}+u\right)$ for some positive constants $m_{1}$ and $m_{2}$. For the other extreme case $d_{1}>0, d_{2}=0$ and $m=1$, Hosono and Ilyas [12] have considered the special case $f(u)=u$ and used the Wazewski principle [10] to show that there always exists a solution for the problem (1.4) - (1.5) with $b=1$ for any $c>0$. Later, Hosono and Ilyas [13] used the shooting argument and the invariant manifold theory to include the case $d_{1}>0, d_{2}>0$ and $m=1$. Recently, Huang [15] has developed a method to treat the problem (1.4) -(1.5) with positive $d_{1}, d_{2}$ and $m=1$ for a general class of functions $f$ which cover all of the models mentioned above. The idea of this method was then used by $\mathrm{Ai}$ and Huang [1] to establish the existence of solutions of the problem (1.4) (1.5) with $d_{2}=0$ and $m=1$ for a class of functions $f$ whose assumption is slightly different from that in 15 .

We note that $m$ is always equal to $1 \mathrm{in}$ all of the above results. The only result (to the authors' knowledge) for the problem (1.4)-(1.5) with $m>1$ is by Hosono [14, where the author considered the special case $d_{1}=0$ and $f(u)=u$ so that the problem (1.4)-(1.5) can be reduced to a planar system and the phase-plane analysis can be employed. On the other hand, this reduction is not available if $d_{1}>0$ and $m>1$. In this paper, we shall develop another means to deal with the problem (1.4)-(1.5) for the extreme case when 
$d_{1}>0, d_{2}=0$ and $m>1$. We shall assume that the function $f$ satisfies the following conditions:

(A1) There exists a unique number $\gamma>0$ such that $f(\gamma)=K$ and

$$
f(u) \in(0, K) \text { for all } u \in(0, \gamma), \quad f(u)>K \text { for all } u \in(\gamma, \infty) .
$$

(A2) $f^{\prime}>0$ on $\left(0, u_{0}\right)$ for some $u_{0} \in(0, \gamma)$ and satisfies

$$
\int_{0^{+}}^{\gamma} \frac{1}{f(u)} d u=\infty
$$

(A3) $\liminf _{u \rightarrow \infty} f(u)>K$.

We first state our main theorem on the existence of traveling waves as follows.

Theorem 1.1. Let $d_{1}>0, d_{2}=0$ and $m>1$. Suppose that $f$ satisfies the assumptions (A1)-(A3). Then, for each given $a \in(0, \gamma)$, there is a nonnegative constant $c(a)$ such that for each $c>c(a)$, there exists a unique solution $(U, V)$ (up to a translation) to (1.4) - 1.5) for some $b=b(a, c) \in(\gamma, \infty)$. Moreover, $U^{\prime}>0$ on $\mathbb{R}, V^{\prime}>0$ on $\left(-\infty, z_{1}\right)$ and $V^{\prime}<0$ on $\left(z_{1},+\infty\right)$, where $z_{1}$ is defined by $U\left(z_{1}\right)=\gamma$. Conversely, for each given $b>\gamma$, there is a nonnegative constant $\bar{c}(b)$ such that for each $c>\bar{c}(b)$, there exists a solution $(U, V)$ to (1.4)-(1.5) for some $a \in(0, \gamma)$.

Next, we have the following theorem on the lower bounds of wave speed.

Theorem 1.2. Let the assumptions of Theorem 1.1 be in force. If $m \in(1,2]$, then $c(a)=0$ for all $a \in(0, \gamma)$ and $\bar{c}(b)=0$ for all $b>\gamma$. If $m>2$, then $c(a)$ is bounded on $(\epsilon, \gamma)$ for each $\epsilon \in(0, \gamma), c(a) \rightarrow 0$ as $a \rightarrow \gamma^{-}$, and $c(a) \rightarrow+\infty$ as $a \rightarrow 0^{+}$.

By a result of Ai and Huang [1] and Theorem 1.2, we see that a traveling wave exists for any positive wave speed if $m \in[1,2]$. We conjecture that the property of zero minimum wave speed should hold for all $m \geq 1$. For $m>2$, due to some technical difficulties, we can only provide here a lower bound estimate for the wave speed as the quantity $c(a)$. We shall leave this important question as an open problem.

We also have the following properties for $b(a, c)$.

Theorem 1.3. Let the assumptions of Theorem 1.1 be in force. Then for each given $a_{0} \in(0, \gamma)$ and $c$ such that $c>c(\hat{a})$ for all $\hat{a} \in\left(a_{0}-\delta_{0}, a_{0}+\delta_{1}\right)$ and for some $\delta_{0} \in\left(0, a_{0}\right)$ and $\delta_{1} \in\left(0, \gamma-a_{0}\right), b(a, c)$ is continuous at $a=a_{0}$. Moreover, it follows that

$$
\begin{aligned}
& \lim _{a \rightarrow \gamma^{-}} b(a, c)=\gamma \text { for each given } c>0, \\
& b(a, c)>a+K \int_{a}^{u_{0}} \frac{1}{f(u)} d u \text { for each } c>c(a),
\end{aligned}
$$

where $u_{0}$ is given in the assumption (A2).

If we further assume that $f$ satisfies the extra assumption:

(A4) $f^{\prime}>0$ on $(0,+\infty)$,

then $b=b(a, c)$ is strictly decreasing in $a \in(0, \gamma)$.

We note that the reaction term $f$ in the existing models [16, 18, 7, 21, 20, 19] satisfies the assumptions (A1)-(A4). Hence our result establishes the existence and uniqueness 
of traveling wave solutions to the reaction-diffusion system associated with the reactions (1.2)-(1.3) (i.e., the system (1.1) with $f(u)=k_{1} u$ and $K=k_{2}$ ) for an isothermal autocatalytic chemical reaction of any order in which the autocatalyst is assumed to decay to the inert product at a rate of the same order.

The method of our proof is based on the method in [1] (see also [15]), where the authors only consider the case $m=1$. However, since we have $m>1$, a solution $\left(U(z), U^{\prime}(z), V(z)\right)$ of the problem (1.4)-(1.5) has to lie on a two-dimensional centre manifold for negative $t$ with $|t| \gg 1$, while the corresponding solution $\left(U(z), U^{\prime}(z), V(z)\right)$ for the case $m=1$ lies on the unique unstable manifold for negative $t$ with $|t| \gg 1$. Therefore, it is nontrivial to establish the local existence and uniqueness of the solution $(U(z), V(z))$ to (1.4) with the condition $\lim _{z \rightarrow-\infty}\left(U(z), U^{\prime}(z), V(z)\right)=(a, 0,0)$ for a given $a \in(0, \gamma)$. The other point is that the growth rate of the $V$ component of a solution $(U, V)$ to (1.4) is faster than the linear rate. Therefore, a possible candidate for a solution of the problem (1.4) -(1.5) may blow up in finite time. Lastly, the $V$ component of a traveling wave solution $(U, V)$ of (1.1) must decay to zero at an algebraic rate, not exponentially fast. Due to these reasons, we need some new techniques and more delicate estimates than those in [1] to overcome these difficulties.

The paper is organized as follows. In section 2, we first use the centre manifold theory to establish the local existence and uniqueness of the solution $(U, V)$ to (1.4) with the condition $\lim _{z \rightarrow-\infty}\left(U, U^{\prime}, V\right)=(a, 0,0)$ for a given $a \in(0, \gamma)$. Then we show that such a solution is globally defined provided $c>c(a)$ for some $c(a) \geq 0$. Finally, in section 3, we establish several auxiliary lemmas to prove Theorems 1.1] 1.3.

\section{Existence of traveling wave solutions.}

2.1. Basic results. Hereafter we shall always assume $m>1$ and $c>0$. Introducing the new variables and parameters

$$
t=\sqrt{d_{1}} z \text { and } \tilde{c}=c / \sqrt{d_{1}},
$$

the system of differential equations (1.4) with $d_{2}=0$ becomes

$$
U^{\prime \prime}+c U^{\prime}-f(U) V^{m}=0, \quad c V^{\prime}+[f(U)-K] V^{m}=0,
$$

which is equivalent to

$$
U^{\prime}=W, \quad W^{\prime}=-c W+f(U) V^{m}, \quad V^{\prime}=\frac{1}{c}[K-f(U)] V^{m},
$$

where for simplicity we have written $\tilde{c}$ as $c$. In order to solve the existence of traveling wave solutions of (1.1), we consider the following (initial value) problem $\left(P_{a}\right)$ :

$$
\begin{aligned}
& \left(\begin{array}{c}
U^{\prime} \\
W^{\prime} \\
V^{\prime}
\end{array}\right)=\left(\begin{array}{c}
W \\
-c W+f(U) V^{m} \\
{[K-f(U)] V^{m} / c}
\end{array}\right), \quad V>0, \\
& \lim _{t \rightarrow-\infty}(U(t), W(t), V(t))=(a, 0,0) .
\end{aligned}
$$

Hereafter, we will let $(U, W, V)$ be a solution of (2.2) satisfying the initial condition

$$
\lim _{t \rightarrow-\infty}(U(t), W(t), V(t))=(a, 0,0)
$$


for a given $a \in(0, \gamma)$. The maximal existence interval of $(U, W, V)$ will be denoted by $(-\infty, T)(T$ may be $+\infty)$. Adding the equations in (2.1) together, we have that

$$
U^{\prime \prime}+c U^{\prime}+c V^{\prime}=K V^{m} \text {. }
$$

Fix $t_{0} \in(-\infty, T)$ and $t \in\left(t_{0}, T\right)$. By a simple computation, it is easy to verify the following equalities (see also [1]):

$$
\begin{aligned}
U^{\prime}(t)= & U^{\prime}\left(t_{0}\right) e^{-c\left(t-t_{0}\right)}+\int_{t_{0}}^{t} e^{-c(t-s)} f(U(s)) V^{m}(s) d s, \\
U(t)= & U\left(t_{0}\right)+\frac{1}{c}\left[1-e^{-c\left(t-t_{0}\right)}\right] U^{\prime}\left(t_{0}\right) \\
& +\frac{1}{c} \int_{t_{0}}^{t}\left[1-e^{-c(t-s)}\right] f(U(s)) V^{m}(s) d s \\
U^{\prime}(t)+c U(t)+c V(t)= & U^{\prime}\left(t_{0}\right)+c U\left(t_{0}\right)+c V\left(t_{0}\right)+K \int_{t_{0}}^{t} V^{m}(s) d s .
\end{aligned}
$$

Letting $t_{0} \rightarrow-\infty$ in (2.7), (2.8) and (2.9), we get

$$
\begin{aligned}
U^{\prime}(t) & =\int_{-\infty}^{t} e^{-c(t-s)} f(U(s)) V^{m}(s) d s \\
U(t) & =a+\frac{1}{c} \int_{-\infty}^{t}\left[1-e^{-c(t-s)}\right] f(U(s)) V^{m}(s) d s, \\
U^{\prime}(t)+c U(t)+c V(t) & =c a+K \int_{-\infty}^{t} V^{m}(s) d s .
\end{aligned}
$$

Also, we have

$$
\begin{aligned}
& V(t)=V\left(t_{0}\right) \exp \left[\frac{1}{c} \int_{t_{0}}^{t}\left[K-f(U(s)) V^{m-1}(s) d s\right]\right], \\
& V(t)=\left(\frac{1}{V^{1-m}\left(t_{0}\right)+(m-1) \int_{t_{0}}^{t}[(f(U(s))-K) / c] d s}\right)^{\frac{1}{m-1}} .
\end{aligned}
$$

Note that (2.14) holds only when $m \neq 1$.

With an elementary calculation, we see that the matrix for the linearized system of (2.3) at the critical point $(a, 0,0)$ with $a>0$ is given by

$$
A_{0}=\left[\begin{array}{ccc}
0 & 1 & 0 \\
0 & -c & 0 \\
0 & 0 & 0
\end{array}\right] .
$$

We note that the resulting linearized system is independent of the order of the reaction $m$ (if $m>1$ ) and the choice of the equilibrium point $(a, 0,0)$. The eigenvalues of $A_{0}$ are $\lambda_{1}=\lambda_{2}=0$ and $\lambda_{3}=-c$, and the corresponding eigenvectors are $(1,0,0)^{t},(0,0,1)^{t}$, and $(1,-c, 0)^{t}$, respectively. Hence (2.3) has a two-dimensional centre manifold at the equilibrium point $(a, 0,0)$ with $a>0$. Moreover, any solution of the problem $\left(P_{a}\right)$ (if it exists) would lie on such a centre manifold.

In the coming subsections, we will discuss the uniqueness and existence of solutions of the problem $\left(P_{a}\right)$. Specifically, for each given $a \in(0, \gamma)$ and $c>0$, we establish 
the uniqueness and local existence of solutions of the problem $\left(P_{a}\right)$ in subsection 2.2; in subsection 2.3, we will show that such a solution can be globally defined provided $c>c(a)$ for some constant $c(a) \geq 0$.

2.2. Local existence and uniqueness of solutions of $\left(P_{a}\right)$. To begin with, we will use the centre manifold theory (cf. 8]) to investigate the local dynamics of the flow of (2.3) near the equilibrium point $(a, 0,0)$. In this subsection, we will always assume $a \in(0, \gamma)$. Define the change of variables

$$
\left(\begin{array}{c}
U-a \\
W \\
V
\end{array}\right)=S_{0}\left(\begin{array}{c}
x \\
y \\
z
\end{array}\right), \quad S_{0}=\left(\begin{array}{ccc}
1 & 0 & 1 \\
0 & 0 & -c \\
0 & 1 & 0
\end{array}\right) .
$$

Then we have

$$
\left(\begin{array}{l}
x \\
y \\
z
\end{array}\right)=\left(\begin{array}{c}
U+\frac{W}{c}-a \\
V \\
-\frac{W}{c}
\end{array}\right),
$$

and the problem $\left(P_{a}\right)$ is reduced to the following (initial value) problem $\left(\hat{P}_{a}\right)$ :

$$
\begin{aligned}
& \left(\begin{array}{c}
x^{\prime} \\
y^{\prime} \\
z^{\prime}
\end{array}\right)=\left(\begin{array}{ccc}
0 & 0 & 0 \\
0 & 0 & 0 \\
0 & 0 & -c
\end{array}\right) \cdot\left(\begin{array}{c}
x \\
y \\
z
\end{array}\right)+\left(\begin{array}{c}
\frac{1}{c} f(x+z+a) y^{m} \\
\frac{1}{c}[K-f(x+z+a)] y^{m} \\
-\frac{1}{c} f(x+z+a) y^{m}
\end{array}\right), \quad y>0, \\
& \lim _{t \rightarrow-\infty}(x(t), y(t), z(t))=(0,0,0) .
\end{aligned}
$$

Moreover, any centre manifold of (2.3) at the equilibrium point $(a, 0,0)$ is transformed into a centre manifold of (2.15) at the origin, whose tangent space is spanned by $(1,0,0)^{t}$ and $(0,1,0)^{t}$, and which can be characterized by a surface

$$
\mathcal{W}^{c}(\mathbf{0}):=\left\{(x, y, z) \in \mathbb{R}^{3} \mid z=\psi(x, y) \text { for }|\mathbf{x}|<\delta_{0}\right\}
$$

for some smooth function $\psi$ and sufficiently small $\delta_{0}$ with $\psi(0,0)=\psi_{x}(0,0)=\psi_{y}(0,0)=$ 0 . Here we denote $\mathbf{x}=(x, y)^{t}$ and $|\mathbf{x}|=\sqrt{x^{2}+y^{2}}$.

For a given $\psi$ which characterizes a centre manifold of (2.15) at the origin, the governing equations of the flow on this centre manifold are given by

$$
x^{\prime}=f(x+\psi(x, y)+a) y^{m} / c, \quad y^{\prime}=[K-f(x+\psi(x, y)+a)] y^{m} / c .
$$

We first study the uniqueness and existence of a solution $(x(t), y(t))$ to (2.17) with the condition $\lim _{t \rightarrow-\infty}(x(t), y(t))=(0,0)$ and $y(t)>0$ for negative $t$ with $|t| \gg 1$. We shall denote this initial value problem as the problem $\left(Q_{a}\right)$. As before, we assume $a \in(0, \gamma)$.

Lemma 2.1. (i) There exists a unique solution $(x(t), y(t)$ ) (up to a translation) of the problem $\left(Q_{a}\right)$ which is defined on $(-\infty, T)$ for some $T \in \mathbb{R} \cup\{+\infty\}$.

(ii) $x>0, y>0, x^{\prime}>0$, and $y^{\prime}>0$ on $(-\infty, T)$.

(iii) For each $\epsilon \in(0, \min \{f(a), K-f(a)\} / 2)$, there exists a negative $t_{\epsilon}$ with $\left|t_{\epsilon}\right| \gg 1$ such that

$$
\begin{aligned}
& C_{1, a,-\epsilon}\left(\frac{y^{1-m}\left(t_{\epsilon}\right)}{C_{a,-\epsilon}(m-1)}+t_{\epsilon}-t\right)^{\frac{1}{1-m}}<x(t)<C_{1, a, \epsilon}\left(\frac{y^{1-m}\left(t_{\epsilon}\right)}{C_{a, \epsilon}(m-1)}+t_{\epsilon}-t\right)^{\frac{1}{1-m}}, \\
& C_{2, a,-\epsilon}\left(\frac{y^{1-m}\left(t_{\epsilon}\right)}{C_{a,-\epsilon}(m-1)}+t_{\epsilon}-t\right)^{\frac{1}{1-m}}<y(t)<C_{2, a, \epsilon}\left(\frac{y^{1-m}\left(t_{\epsilon}\right)}{C_{a, \epsilon}(m-1)}+t_{\epsilon}-t\right)^{\frac{1}{1-m}}
\end{aligned}
$$


for $t \leq t_{\epsilon}$, where

$$
\begin{aligned}
C_{1, a, \pm \epsilon} & :=\left(\frac{m-1}{c}\right)^{\frac{1}{1-m}}(f(a) \pm \epsilon)(K-f(a) \pm \epsilon)^{\frac{m}{1-m}} \\
C_{2, a, \pm \epsilon} & :=\left(\frac{m-1}{c}\right)^{\frac{1}{1-m}}(K-f(a) \pm \epsilon)^{\frac{1}{1-m}} \\
C_{a, \pm \epsilon} & :=(K-f(a) \pm \epsilon) / c .
\end{aligned}
$$

(iv) Let $\left(x_{a_{i}}, y_{a_{i}}\right), i=1,2$, be the solution of $\left(Q_{a_{i}}\right)$ with $a_{1}, a_{2} \in(0, \gamma)$ and $a_{2}>a_{1}$. If $f^{\prime}>0$ on $(0, \gamma)$, then $y_{a_{2}}(t)>y_{a_{1}}(t)$ for negative $t$ with $|t| \gg 1$.

(v) Let $\left(U_{a_{i}}, V_{a_{i}}\right), i=1,2$, be the solution of $\left(P_{a_{i}}\right)$ with $a_{1}, a_{2} \in(0, \gamma)$ and $a_{2}>a_{1}$. If $f^{\prime}>0$ on $(0, \gamma)$, then $V_{a_{2}}(t)>V_{a_{1}}(t)$ for negative $t$ with $|t| \gg 1$.

Proof. To solve the problem $\left(Q_{a}\right)$, it suffices to consider the following initial value problem $\left(\widehat{Q}_{a}\right)$ :

$$
\frac{d y}{d x}=\frac{K-f(x+\psi(x, y)+a)}{f(x+\psi(x, y)+a)}, \quad y(0)=0 .
$$

Recall that $\psi(x, y)=o(|\mathbf{x}|)$. Here we write $\mathbf{x}=(x, y)$ and $|\mathbf{x}|=\sqrt{x^{2}+y^{2}}$. Hence if $x$ and $y$ are sufficiently small, the above problem is equivalent to the problem

$$
\frac{d y}{d x}=\frac{K-f(a)}{f(a)}+N(x, y), \quad y(0)=0
$$

where $N(x, y)$ is smooth in $x$ and $y$ and $O\left(\sqrt{x^{2}+y^{2}}\right)$. Therefore, by the standard theory of differential equations, we can conclude that there exists a unique solution $y(x)$ of $\left(\widehat{Q}_{a}\right)$ defined on $\left[0, x_{0}\right]$ for some sufficiently small $x_{0}>0$ such that $y(x)>0$, $K-f(x+\psi(x, y(x))+a)>(K-f(a)) / 2$, and $f(x+\psi(x, y(x))+a)>f(a) / 2$ for all $x \in\left[0, x_{0}\right]$. Using this and (2.17), the assertions (i) and (ii) follow.

To investigate the asymptotical behavior of $(x(t), y(t))$, we first note that (2.17) is reduced to the following system:

$$
\begin{aligned}
x^{\prime} & =\left(f(a)+O(|\mathbf{x}|) y^{m} / c,\right. \\
y^{\prime} & =\left(K-f(a)+O(|\mathbf{x}|) y^{m} / c,\right.
\end{aligned}
$$

for all $x$ and $y$ sufficiently small. Hence for each given $\epsilon \in(0, \min \{f(a), K-f(a)\} / 2)$, we can choose a negative $t_{\epsilon}$ with $\left|t_{\epsilon}\right| \gg 1$ such that

$$
\begin{aligned}
\frac{f(a)-\epsilon}{c} y^{m}(t) & <x^{\prime}(t)<\frac{f(a)+\epsilon}{c} y^{m}(t), \\
\frac{K-f(a)-\epsilon}{c} y^{m}(t) & <y^{\prime}(t)<\frac{K-f(a)+\epsilon}{c} y^{m}(t)
\end{aligned}
$$

holds for $t \leq t_{\epsilon}$. Now by integrating the inequality (2.21) from $-\infty$ to $t_{\epsilon}$, we see that (2.19) holds. Then by substituting (2.19) into (2.20) and integrating the resulting inequality from $-\infty$ to $t_{\epsilon}$, we then obtain (2.18). This establishes the assertion (iii).

Now we turn to the proof of the assertion (iv). Since $f^{\prime}>0$ on $(0, \gamma)$, we can fix a sufficiently small $\epsilon_{0} \in\left(0, \min _{i=1,2}\left\{f\left(a_{i}\right), K-f\left(a_{i}\right)\right\} / 2\right)$ such that $C_{1, a_{2},-\epsilon_{0}}>C_{1, a_{1}, \epsilon_{0}}$ and 
$C_{2, a_{2},-\epsilon_{0}}>C_{2, a_{1}, \epsilon_{0}}$. By the assertion (iii), we can choose a negative $t_{\epsilon_{0}}$ with $\left|t_{\epsilon_{0}}\right| \gg 1$ such that

$C_{2, a_{i},-\epsilon_{0}}\left(\frac{y_{a_{i}}^{1-m}\left(t_{\epsilon_{0}}\right)}{C_{a_{i},-\epsilon_{0}}(m-1)}+t_{\epsilon_{0}}-t\right)^{\frac{1}{1-m}}<y_{a_{i}}(t)<C_{2, a_{i}, \epsilon_{0}}\left(\frac{y_{a_{i}}^{1-m}\left(t_{\epsilon_{0}}\right)}{C_{a_{i}, \epsilon_{0}}(m-1)}+t_{\epsilon_{0}}-t\right)^{\frac{1}{1-m}}$

hold for $t \leq t_{\epsilon_{0}}$ and $i=1,2$. Together with the choices of $C_{i, a_{i}, \pm \epsilon_{0}}, i=1,2$, we can conclude that $y_{a_{2}}(t)>y_{a_{1}}(t)$ for all $t \leq t_{1}$ and for some $t_{1}<t_{\epsilon_{0}}$.

Finally, with the help of the assertion (iv) and the relationship between the variables $(x, y)$ and $(U, V)$, we then obtain the assertion (v). The proof is thus completed.

We shall find the asymptotic expansion of any centre manifold. To this end, we first note that a centre manifold of (2.15) at the origin characterized by $\psi$ satisfies the relation $($ see $[8])$ :

$$
\begin{aligned}
& -c \psi(x, y)-f(x+\psi(x, y)+a) \frac{y^{m}}{c} \\
= & \psi_{x}(x, y) f(x+\psi(x, y)+a) \frac{y^{m}}{c}+\psi_{y}(x, y)[K-f(x+\psi(x, y)+a)] \frac{y^{m}}{c}
\end{aligned}
$$

for all $\mathbf{x}$ with $|\mathbf{x}|<\delta_{0}$ and some $\delta_{0}>0$. Since this is an identity for all $\mathbf{x}$ with $|\mathbf{x}|<\delta_{0}$, we then obtain

$$
\psi(x, y)=-(f(a)+O(|\mathbf{x}|)) \frac{y^{m}}{c^{2}} .
$$

Notice that the leading term in (2.22) is independent of $\psi$.

Lemma 2.2. For each $c>0$ and $a \in(0, \gamma)$, there exists a unique solution $(U, V)$ (up to a translation) of (2.1) which is defined on $(-\infty, T)$ for some $T \in \mathbb{R} \cup\{+\infty\}$, such that the following hold:

(i) $U(t)>a, U^{\prime}(t)>0$, and $V(t)>0$ for all $t \in(-\infty, T)$.

(ii) $V^{\prime}(t)(\gamma-U(t))>0$ for $U(t) \neq \gamma$.

(iii) $\lim _{t \rightarrow-\infty}\left(U(t), U^{\prime}(t), V(t)\right)=(a, 0,0)$.

(iv) $(U, W, V)=\left(U(t), U^{\prime}(t), V(t)\right)$ lies on a surface $W=\Psi(U, V)$ for negative $t$ with $|t| \gg 1$ which takes the form

$$
W=\left(\frac{f(a)}{c}+O\left(\sqrt{(U-a)^{2}+V^{2}}\right)\right) \cdot V^{m} .
$$

Proof. Take any $\psi$ which characterizes a centre manifold of (2.15) at the origin. From Lemma 2.1 it follows that there exists a unique solution $(x(t), y(t))$ (up to a translation) of (2.17) (with respect to this given $\psi$ ) defined for all $t \in(-\infty, 0]$ (by a suitable shift of $t$ ) such that $x>0, y>0, x^{\prime}>0$, and $y^{\prime}>0$ on $(-\infty, 0]$, and $\lim _{t \rightarrow-\infty}(x(t), y(t))=(0,0)$. Therefore, by (2.22), there exists a solution $(x(t), y(t), z(t))$ of the problem $\left(\hat{P}_{a}\right)$ defined on $(-\infty, 0]$ such that $x>0, y>0, z<0, x^{\prime}>0$, and $y^{\prime}>0$ on $(-\infty, 0]$, and $\lim _{t \rightarrow-\infty}(x(t), y(t), z(t))=(0,0,0)$.

Next, we want to show the uniqueness of the solution to $\left(\hat{P}_{a}\right)$. We will use the method of [2] to prove it. Since any solution of $\left(\hat{P}_{a}\right)$ must lie on a centre manifold of (2.15) at $(0,0,0)$, it suffices to show that $(x(t), y(t), z(t))$ is independent of the choice of $\psi$. Suppose that $\psi_{i}, i=1,2$, are the representations of two centre manifolds of (2.15) at $(0,0,0)$. Let $(x(t), y(t))$ be the solution of (2.17) with $\psi=\psi_{1}$ obtained above. Then 
$(x(t), y(t), z(t)):=\left(x(t), y(t), \psi_{1}(x(t), y(t))\right)$ is a solution of the problem $\left(\hat{P}_{a}\right)$ defined on $(-\infty, 0]$.

Note that for negative $t$ with $|t| \gg 1,\left(x(t), y(t), \psi_{2}(x(t), y(t))\right)$ lies on the centre manifold associated with $\psi_{2}$. We claim that $z(t)=\psi_{2}(x(t), y(t))$ for negative $t$ with $|t| \gg 1$. To this end, we claim that $\eta(t):=z(t)-\psi_{2}(x(t), y(t))=0$ for negative $t$ with $|t| \gg 1$. Note that $\eta(t)$ is bounded. Recall that the centre manifold $\left(x, y, \psi_{2}(x, y)\right)$ satisfies

$$
\psi_{2, x} f\left(x+\psi_{2}+a\right) \frac{y^{m}}{c}+\psi_{2, y}\left[K-f\left(x+\psi_{2}+a\right)\right] \frac{y^{m}}{c}=-c \psi_{2}-f\left(x+\psi_{2}+a\right) \frac{y^{m}}{c}
$$

for all sufficiently small $x, y$. Here, for simplicity, we write $\partial \psi_{2} / \partial x$ and $\partial \psi_{2} / \partial y$ as $\psi_{2, x}$ and $\psi_{2, y}$, respectively, and ignore the arguments of $\psi_{2}, \psi_{2, x}, \psi_{2, y}$. Also note that $z(t)$ satisfies

$$
z^{\prime}(t)=-c z(t)-f(x(t)+z(t)+a) \frac{y^{m}(t)}{c}
$$

for all $t \in(-\infty, 0]$. Subtracting (2.23) from this equation, we obtain

$$
\begin{aligned}
\eta^{\prime}(t)= & -c \eta(t)-\left[f(x(t)+z(t)+a)-f\left(x(t)+\psi_{2}(x(t), y(t))+a\right)\right] \frac{y^{m}(t)}{c} \\
& +\psi_{2, x}(x(t), y(t))\left[f\left(x(t)+\psi_{2}(x(t), y(t))+a\right)-f(x(t)+z(t)+a)\right] \frac{y^{m}(t)}{c} \\
& +\psi_{2, y}(x(t), y(t))\left[f(x(t)+z(t)+a)-f\left(x(t)+\psi_{2}(x(t), y(t))+a\right)\right] \frac{y^{m}(t)}{c}
\end{aligned}
$$

for negative $t$ with $|t| \gg 1$. It follows from the mean-value theorem that

$$
\eta^{\prime}(t)=-c \eta(t)+M(t) y^{m}(t) \eta(t)
$$

for negative $t$ with $|t| \gg 1$ and for some bounded function $M(t)$. Solving the above equation and using $\eta(-\infty)=0$, we have

$$
\eta(t)=\int_{-\infty}^{t} e^{-c(t-s)} M(s) y^{m}(s) \eta(s) d s
$$

for negative $t$ with $|t| \gg 1$. Since $M(t)$ is bounded and $y(t) \rightarrow 0$ as $t \rightarrow-\infty$, there exists a negative $s_{0}$ with $\left|s_{0}\right| \gg 1$ such that $\left|M(s) y^{m}(s)\right| \leq c / 2$ for all $s \in\left(-\infty, s_{0}\right]$. Together with the above equation, this yields

$$
\begin{aligned}
|\eta(t)| & \leq \frac{c}{2} \int_{-\infty}^{t} e^{-c(t-s)}|\eta(s)| d s \\
& \leq \frac{1}{2} \sup _{s \in\left(-\infty, s_{0}\right]}|\eta(s)|
\end{aligned}
$$

for all $t \in\left(-\infty, s_{0}\right]$. Hence we have $\eta(t)=0$ for all $t \in\left(-\infty, s_{0}\right]$.

Therefore, $\left.z(t)=\psi_{2}(x(t), y(t))\right)$ for negative $t$ with $|t| \gg 1$ and so $(x(t), y(t))$ is also a solution of (2.17) with $\psi=\psi_{2}$ satisfying the condition $\lim _{t \rightarrow-\infty}(x(t), y(t))=(0,0)$. Then, by part (i) of Lemma 2.1. we obtain the uniqueness solution to $\left(\hat{P}_{a}\right)$.

Finally, transferring $(U, W, V)$ back to the origin, we can conclude that there exists a unique solution $(U(t), W(t), V(t))$ (up to a translation) of the problem $\left(P_{a}\right)$ defined on the maximal existence interval $(-\infty, T)$ such that $U>a, U^{\prime}=W>0, V>0$ for negative $t$ with $|t| \gg 1$, and $\lim _{t \rightarrow-\infty}\left(U(t), U^{\prime}(t), V(t)\right)=(a, 0,0)$. Furthermore, with 
the help of the third equation in (2.2), (2.10), (2.11), and (2.13), we have that $U>a$, $U^{\prime}>0, V>0$ on $(-\infty, T)$, and $V^{\prime}(t)(\gamma-U(t))>0$ for $U(t) \neq \gamma$. Also, statement (iv) follows from (2.22). This proves the lemma.

2.3. Global existence of solutions of $\left(P_{a}\right)$. For each $a \in(0, \gamma)$ and $c>0$, the unique solution $(U, V)$ of (2.1) obtained in Lemma 2.2 is a good candidate for a solution to (1.4) -(1.5). However, since $m>1$, from the third equation in (2.2) we see that $(U, V)$ may blow up in finite time. In this subsection, we will show that such a solution is globally defined if $c$ is suitably chosen.

First we observe the following lemma.

Lemma 2.3. Let $(U, W, V)$ be a solution of $\left(P_{a}\right)$ defined on $(-\infty, T)$. If $V$ is bounded on $(-\infty, T)$, then we have $T=+\infty$.

Proof. The proof is a slight modification of that of [1, part (i) of Lemma 2.1]. Fix a $t_{0} \in(-\infty, T)$. Using Lemma 2.2 and the boundedness of $V$, there exists a positive constant $M$ such that $U>a, U^{\prime}>0$, and $V^{m-1} \in(0, M / K)$ on $(-\infty, T)$. Therefore from (2.9) it follows that for all $t \in\left(t_{0}, T\right)$,

$$
\begin{aligned}
U^{\prime}(t)+c U(t)+c V(t) & =U^{\prime}\left(t_{0}\right)+c U\left(t_{0}\right)+c V\left(t_{0}\right)+K \int_{t_{0}}^{t} V^{m}(s) d s \\
& \leq U^{\prime}\left(t_{0}\right)+c U\left(t_{0}\right)+c V\left(t_{0}\right)+M \int_{t_{0}}^{t} V(s) d s \\
& \leq U^{\prime}\left(t_{0}\right)+c U\left(t_{0}\right)+c V\left(t_{0}\right)+\frac{M}{c} \int_{t_{0}}^{t}\left(U^{\prime}(s)+c U(s)+c V(s)\right) d s,
\end{aligned}
$$

which together with an application of Gronwall's inequality yields

$$
U^{\prime}(t)+c U(t)+c V(t) \leq\left[U^{\prime}\left(t_{0}\right)+c U\left(t_{0}\right)+c V\left(t_{0}\right)\right] e^{M\left(t-t_{0}\right) / c}
$$

for all $t \in\left(t_{0}, T\right)$. Recall that $U>a, U^{\prime}>0$, and $V>0$ on $(-\infty, T)$. The above inequality implies $T=+\infty$. This completes the proof.

Now we come to the goal of this subsection.

Lemma 2.4. Let $(U, W, V)$ be a solution of $\left(P_{a}\right)$ defined on $(-\infty, T)$. Then there exists a constant $c(a) \geq 0$ such that $T=+\infty$ if $c>c(a)$. Moreover, if $m \in(1,2]$, then $c(a)=0$ for all $a \in(0, \gamma)$; and if $m>2$, then $c(a)$ is bounded on $(\epsilon, \gamma)$ for each $\epsilon \in(0, \gamma), c(a) \rightarrow 0$ as $a \rightarrow \gamma^{-}$, and $c(a) \rightarrow+\infty$ as $a \rightarrow 0^{+}$.

Proof. We first define the quantity $c(a)$. Note that $L(a):=\min _{u \in[a, \gamma]} f(u)$ is a welldefined positive constant for each $a \in(0, \gamma)$, and $\lim _{a \rightarrow 0^{+}} L(a)=0$. Set $l(n):=\inf \{k \in$ $(0,+\infty) \mid k x \geq x^{m}$ for all $\left.x \in[0, n]\right\}$. Note that $l(n)=n^{m-1}$. Now we consider the case $m>2$. There exists a unique least positive integer $n_{0}=n_{0}(a) \in \mathbb{N}$ such that

$$
n_{0}>\frac{\gamma\left(\max _{s \in[a, \gamma]}(K-f(s))\right)}{L(a)} .
$$


Notice that $n_{0}$ is decreasing in $a \in(0, \gamma), n_{0}=1$ for $a \in(0, \gamma)$ with $|\gamma-a| \ll 1$, and $n_{0} \nearrow+\infty$ as $a \searrow 0^{+}$. Note that the function

$$
h_{n}: c \longrightarrow \frac{c}{\frac{m K}{c} \cdot l(n)+c}
$$

is increasing on $(0,+\infty)$ such that $\lim _{c \rightarrow 0^{+}} h_{n}(c)=0$ and $\lim _{c \rightarrow+\infty} h_{n}(c)=1$ for each $n \in \mathbb{N}$. Hence we can choose a positive number $c(a)$ such that

$$
\frac{c(a)}{\frac{m K}{c(a)} \cdot l\left(n_{0}\right)+c(a)}=\frac{1}{n_{0}} \cdot \frac{\gamma\left(\max _{s \in[a, \gamma]}(K-f(s))\right)}{L(a)} .
$$

It is easy to see that $c(a) \rightarrow 0$ as $a \rightarrow \gamma^{-}$, and $c(a)$ is bounded on $(\epsilon, \gamma)$ for each $\epsilon \in(0, \gamma)$. Furthermore, from $L\left(0^{+}\right)=0$ it follows that $c(a) \rightarrow+\infty$ as $a \rightarrow 0^{+}$. Notice that $h_{n} \geq h_{n+1}$ and $h_{n}$ is increasing on $(0,+\infty)$ for each $n \in \mathbb{N}$. Then with the choice of $n_{0}$ and $c(a)$, we have

$$
\sum_{n=1}^{n_{0}} \frac{c L(a)}{\left(\frac{m K}{c} \cdot l(n)+c\right) \cdot\left(\max _{s \in[a, \gamma]}(K-f(s))\right)} \geq \gamma
$$

for all $c \geq c(a)$. On the other hand, if $m \in(1,2]$, we define $c(a)=0$ for each $a \in(0, \gamma)$. Note that $\sum_{n=1}^{\infty} \frac{1}{l(n)}=+\infty$ for $m \in(1,2]$. Hence for each fixed $m \in(1,2], c>0$ and $a \in(0, \gamma)$, we can choose a sufficiently large $n_{0}=n_{0}(a, c, m) \in \mathbb{N}$ such that (2.24) holds.

Now we will show that the solution $(U, W, V)$ of $\left(P_{a}\right)$ is globally defined, if $c>c(a)$. Suppose on the contrary that $T<+\infty$. Note that from the third equation in (2.2), we have $V^{\prime}(t)<0$ if $U(t)>\gamma$. Hence from Lemma 2.3 and parts (i) and (ii) of Lemma 2.2. it follows that $V(t) \nearrow+\infty$ and $U(t) \nearrow U_{0}$ as $t \rightarrow T^{-}$for some $U_{0} \in(a, \gamma]$. Also note that $V(-\infty)=0$.

Set $s_{n}:=\sup \{s \in(-\infty, T) \mid V(t)<n$ for all $t \in(-\infty, s]\}$ for each $n \in \mathbb{N}$. Then $s_{n}$ is well-defined for each $n \in \mathbb{N}$, and $s_{i}<s_{j}$ if $i<j$. It follows from (2.2) that for each $n \in \mathbb{N}$ and for all $t \in\left(-\infty, s_{n}\right]$,

$$
\begin{aligned}
\left(\frac{W}{V^{m}}\right)^{\prime} & =-m\left(\frac{W}{V^{m+1}}\right) V^{\prime}+\frac{W^{\prime}}{V^{m}} \\
& =-\frac{m(K-f(U))}{c}\left(\frac{W}{V}\right)+\left(-c \cdot \frac{W}{V^{m}}+f(U)\right) \\
& \geq-\frac{m(K-f(U))}{c}\left(\frac{W}{V^{m} / l(n)}\right)+\left(-c \cdot \frac{W}{V^{m}}+f(U)\right),
\end{aligned}
$$

by using the definitions of $s_{n}$ and $l(n)$. Hence we have

$$
\left(\frac{W}{V^{m}}\right)^{\prime} \geq-\left(\frac{m K}{c} \cdot l(n)+c\right) \frac{W}{V^{m}}+f(U) .
$$

Recall from part (iv) of Lemma 2.2 that $\lim _{t \rightarrow-\infty} W(t) / V^{m}(t)=f(a) / c$. Then an integration of the above inequality gives

$$
\begin{aligned}
\frac{W(t)}{V^{m}(t)} & \geq \int_{-\infty}^{t} e^{-\left[\frac{m K}{c} \cdot l(n)+c\right](t-s)} f(U(s)) d s \\
& \geq L(a) /\left[\frac{m K}{c} \cdot l(n)+c\right]
\end{aligned}
$$


for all $t \in\left(-\infty, s_{n}\right]$. This, together with the third equation in (2.2), yields

$$
\begin{aligned}
U^{\prime}(t)=W(t) & \geq \frac{L(a)}{\frac{m K}{c} \cdot l(n)+c} \cdot \frac{c V^{\prime}(t)}{K-f(U(t))} \\
& \geq \frac{c L(a)}{\frac{m K}{c} \cdot l(n)+c} \cdot \frac{V^{\prime}(t)}{\max _{s \in[a, \gamma]}(K-f(s))}
\end{aligned}
$$

for all $t \in\left(-\infty, s_{n}\right]$. Integrating the above inequality over $\left[s_{n-1}, s_{n}\right]$ with $s_{0}=-\infty$, we then have

$$
U\left(s_{n}\right)-U\left(s_{n-1}\right) \geq \frac{c L(a)}{\left(\frac{m K}{c} \cdot l(n)+c\right) \cdot\left(\max _{s \in[a, \gamma]}(K-f(s))\right)}
$$

for all $n=1, \ldots, n_{0}$. Summing this inequality from $n=1$ to $n_{0}$, we then obtain

$$
U\left(s_{n_{0}}\right)-a \geq \sum_{n=1}^{n_{0}} \frac{c L(a)}{\left(\frac{m K}{c} \cdot l(n)+c\right) \cdot\left(\max _{s \in[a, \gamma]}(K-f(s))\right)} .
$$

This is a contradiction to (2.24), thereby completing the proof of this lemma.

3. Proofs of main results. Throughout this section, we will let $(U(t ; a, c)$, $W(t ; a, c), V(t ; a, c))$ be a unique solution of $\left(P_{a}\right)$ with $c>c(a)$ defined on $(-\infty, \infty)$, which was shown to exist in Section 2. We also denote by $b(a, c)$ the limit $\lim _{t \rightarrow+\infty} U(t ; a, c)$, which will be shown to exist in Lemma 3.1. If there is no ambiguity, we will write $(U(t ; a, c), W(t ; a, c), V(t ; a, c))$ and $b(a, c)$ as $(U(t), W(t), V(t))$ and $b$, respectively. Before giving the proofs of Theorems 1.1 1.3, we need some preparation.

3.1. Auxiliary lemmas.

Lemma 3.1. For $a \in(0, \gamma),\left(U, U^{\prime}, V\right)$ satisfies the following:

(i) there exists a $t_{1} \in \mathbb{R}$ such that $U\left(t_{1}\right)=\gamma, V^{\prime}>0$ on $\left(-\infty, t_{1}\right)$ and $V^{\prime}<0$ on $\left(t_{1}, \infty\right)$;

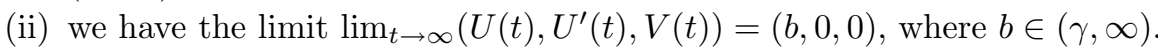

Proof. First, we consider part (i). The proof of part (i) is similar to that of [1, part (ii) of Lemma 2.1]. Since the argument is short, we sketch it here for the convenience of the reader.

Set $t_{1}:=\sup \{t \in \mathbb{R}: U<\gamma$ on $(-\infty, t]\}$. Since $U(t) \in(a, \gamma)$ for negative $t$ with $|t| \gg 1$, we can choose a $t_{0}$ such that $U<\gamma$ on $\left(-\infty, t_{0}\right]$, and set

$$
\hat{t}_{1}:=t_{0}+\frac{1}{c}+\frac{\gamma-a}{k_{1} V^{m}\left(t_{0}\right)}, \quad k_{1}:=\min \left\{\frac{f(u)}{c}: U\left(t_{0}\right) \leq u \leq \gamma\right\} .
$$

Now we claim that $t_{1} \leq \hat{t}_{1}$. For a contradiction, we assume that $t_{1}>\hat{t}_{1}$. Then we have $U(t)<\gamma$ for all $t \in\left(t_{0}, \hat{t}_{1}\right]$. From parts (i) and (ii) of Lemma 2.2] it follows that $U^{\prime}\left(t_{0}\right)>0, V^{\prime}(t)>0$ and $V(t)>V\left(t_{0}\right)$ for all $t \in\left(t_{0}, \hat{t}_{1}\right]$. These facts, together with 
(2.8) and the choice of $\hat{t}_{1}$, give

$$
\begin{aligned}
U\left(\hat{t}_{1}\right) & >a+k_{1} V^{m}\left(t_{0}\right) \int_{t_{0}}^{\hat{t}_{1}}\left[1-e^{-c\left(\hat{t}_{1}-s\right)}\right] d s \\
& =a+k_{1} V^{m}\left(t_{0}\right)\left[\hat{t}_{1}-t_{0}-\frac{1-e^{-c\left(\hat{t}_{1}-t_{0}\right)}}{c}\right]>\gamma,
\end{aligned}
$$

a contradiction. Hence $t_{1} \leq \hat{t}_{1}<+\infty$. By using the continuity of $U$, we then have that $U\left(t_{1}\right)=\gamma$. The assertion for $V$ then follows from part (ii) of Lemma 2.2

Now we consider part (ii). Fix $t_{2} \in\left(t_{1}, \infty\right)$ and set

$$
\rho:=\inf \left\{\frac{f(u)-K}{c}: u \in\left[U\left(t_{2}\right), \infty\right)\right\},
$$

which is positive by assumptions (A1) and (A3). By using (2.14) with $t_{0}=t_{2}$, we have that

$$
V(t) \leq\left(\frac{1}{V^{1-m}\left(t_{2}\right)+(m-1) \rho\left(t-t_{2}\right)}\right)^{\frac{1}{m-1}}
$$

for all $t \geq t_{2}$. Hence $V(t) \rightarrow 0$ as $t \rightarrow \infty$. Furthermore, since $m /(m-1)>1$, there exists a positive constant $K_{1}$ such that for all $t \in\left[t_{2}, \infty\right)$, it follows that

$$
\int_{t_{2}}^{t} V^{m}(s) d s \leq \int_{t_{2}}^{t}\left(\frac{1}{V^{1-m}\left(t_{2}\right)+(m-1) \rho\left(s-t_{2}\right)}\right)^{\frac{m}{m-1}} d s \leq K_{1} .
$$

Together with (2.12), this yields

$$
U^{\prime}(t)+c U(t)+c V(t) \leq c a+K \int_{-\infty}^{t_{2}} V^{m}(s) d s+K K_{1}
$$

for all $t \geq t_{2}$. Recall that $U, U^{\prime}$ and $V$ are positive on $\mathbb{R}$. Then the above inequality implies that $U$ is bounded above. Hence $U(t) \nearrow b$ as $t \rightarrow+\infty$ for some $b=b(a, c) \in$ $(\gamma, \infty)$.

Now we will show that $U^{\prime}(t) \rightarrow 0$ as $t \rightarrow+\infty$. To this end, we note that the function

$$
h: t \rightarrow \int_{-\infty}^{t} e^{c s} f(U(s)) V^{m}(s) d s
$$

is increasing on $\mathbb{R}$. Therefore, $h(+\infty):=\lim _{t \rightarrow+\infty} h(t)$ exists. If $h(+\infty)$ is finite, then by applying (2.10), we obtain

$$
\lim _{t \rightarrow+\infty} U^{\prime}(t)=\lim _{t \rightarrow+\infty} e^{-c t} h(t)=0 .
$$

If $h(+\infty)=+\infty$, then by using (2.10) and l'Hôpital's rule, this yields

$$
\lim _{t \rightarrow+\infty} U^{\prime}(t)=\lim _{t \rightarrow+\infty} \frac{h(t)}{e^{c t}}=\lim _{t \rightarrow+\infty} f(U(t)) V^{m}(t) / c=0 .
$$

Hence $U^{\prime}(t) \rightarrow 0$ as $t \rightarrow+\infty$ in any case. This completes the proof.

Lemma 3.2. Let $t_{1}$ be such that $U\left(t_{1} ; a, c\right)=\gamma$. The following statements hold:

(i) For each given $c>0$, there exists a $\delta_{0} \in(0,1 / 2)$ such that if $a \in(\gamma-\delta, \gamma)$ with $\delta \in\left(0, \delta_{0}\right]$, we have $V\left(t_{1} ; a, c\right) \leq \delta$.

(ii) For each given $c>0, \lim _{a \rightarrow \gamma^{-}} b(a, c)=\gamma$. 
Proof. We first prove (i). Let

$$
M:=\sup _{s \in[\gamma / 2, \gamma]}\left|f^{\prime}(s)\right| \text { and } \delta_{1}:=\min \left\{\frac{\left(2^{m-1}-1\right) K}{2^{m+2}(m-1) M},\left(\frac{\left(2^{m-2}-1 / 2\right) c^{2}}{(m-1) M}\right)^{1 / m}\right\} .
$$

Note that $f(\gamma)=K$ and $\lim _{a \rightarrow \gamma^{-}} c(a)=0$ by Lemma 2.4. Therefore we can choose a $\delta_{0} \in\left(0, \min \left\{\gamma / 4, \delta_{1}, 1 / 2\right\}\right)$ such that $f(u) \geq K / 2$ for all $u \in\left[\gamma-\delta_{0}, \gamma\right]$, and $c>c(a)$ for all $a \in\left(\gamma-\delta_{0}, \gamma\right)$. In the remainder of the proof, for simplicity, we will write $(U(t ; a, c), W(t ; a, c), V(t ; a, c))$ as $(U(t), W(t), V(t))$. We will show that this $\delta_{0}$ satisfies the required property. For this, we fix a $\delta \in\left(0, \delta_{0}\right]$ and $a \in(\gamma-\delta, \gamma)$. Since $\delta / 2<\delta$, it suffices to prove the case when $V\left(t_{1}\right)>\delta / 2$. To begin with, since $V^{\prime}>0$ on $\left(-\infty, t_{1}\right)$, we can choose a $t_{0} \in\left(-\infty, t_{1}\right)$ with the property that $V\left(t_{0}\right)=\delta / 2$. Taking into account the fact that $U(t) \in(\gamma-\delta, \gamma)$ and $V^{\prime}(t)>0$ for all $t \in\left[t_{0}, t_{1}\right)$, we have that $f(U(t)) V^{m}(t) \geq$ $K \delta^{m} / 2^{m+1}$ for all $t \in\left[t_{0}, t_{1}\right]$. Together with (2.8) and $U^{\prime}\left(t_{0}\right)>0$, this yields

$$
\begin{aligned}
U\left(t_{1}\right) & =U\left(t_{0}\right)+\frac{1}{c}\left[1-e^{-c\left(t_{1}-t_{0}\right)}\right] U^{\prime}\left(t_{0}\right)+\frac{1}{c} \int_{t_{0}}^{t_{1}}\left[1-e^{-c\left(t_{1}-s\right)}\right] f(U(s)) V^{m}(s) d s \\
& >U\left(t_{0}\right)+\frac{K \delta^{m}}{2^{m+1} c}\left(t_{1}-t_{0}-\frac{1}{c}\right),
\end{aligned}
$$

which implies

$$
\begin{aligned}
t_{1}-t_{0} & <\frac{2^{m+1} c}{K \delta^{m}}\left[U\left(t_{1}\right)-U\left(t_{0}\right)\right]+\frac{1}{c} \\
& <\frac{2^{m+1} c}{K \delta^{m}}(\gamma-a)+\frac{1}{c} \\
& <\frac{2^{m+1} c}{K} \delta^{1-m}+\frac{1}{c} \quad(\text { since } a \in(\gamma-\delta, \gamma)) .
\end{aligned}
$$

With the help of (2.14) and the above estimate on $t_{1}-t_{0}, V\left(t_{1}\right)$ can be estimated as follows:

$$
\begin{aligned}
V^{1-m}\left(t_{1}\right) & =V^{1-m}\left(t_{0}\right)+(m-1) \int_{t_{0}}^{t_{1}}[(f(U(s))-K) / c] d s \\
& \geq\left(\frac{\delta}{2}\right)^{1-m}-\frac{m-1}{c} \cdot \sup _{s \in[\gamma / 2, \gamma]}\left|f^{\prime}(s)\right| \cdot \sup _{s \in\left[t_{0}, t_{1}\right]}|U(s)-\gamma| \cdot\left(t_{1}-t_{0}\right)
\end{aligned}
$$

(by the mean-value theorem and $f(\gamma)=K$ )

$$
\geq\left(\frac{\delta}{2}\right)^{1-m}-\frac{m-1}{c} \cdot M \delta \cdot\left(\frac{2^{m+1} c}{K} \delta^{1-m}+\frac{1}{c}\right)
$$

(since $U(t) \in(\gamma-\delta, \gamma)$ for all $t \in\left[t_{0}, t_{1}\right)$ )

$$
\begin{aligned}
\geq & \left(2^{m-1}-\frac{(m-1) M 2^{m+1}}{K} \delta\right) \delta^{1-m}-\frac{(m-1) M}{c^{2}} \delta \\
\geq & \left(2^{m-1}-\left(2^{m-1}-1\right) / 2\right) \delta^{1-m}-\frac{(m-1) M}{c^{2}} \delta \\
& \left(\text { since } \delta<\left(2^{m-1}-1\right) K /\left[2^{m+2}(m-1) M\right]\right) \\
\geq & \delta^{1-m} \quad\left(\text { since } \delta<\delta_{1}\right) .
\end{aligned}
$$

This gives $V\left(t_{1}\right) \leq \delta$, thereby completing the proof of (i). 
Now we turn to show (ii). Given a sufficiently small $\epsilon>0$, it suffices to show that there exists a $\delta>0$ such that $b(a, c) \in(\gamma, \gamma+\epsilon]$ if $a \in(\gamma-\delta, \gamma)$. We first give the definition of the required $\delta$. Indeed, we choose a $p>1$ such that $m /[p(m-1)]>1$, and let $q>1$ satisfy $1 / p+1 / q=1$. Define

$$
\delta:=\min \left\{\delta_{0}, \frac{c^{2} \epsilon}{4 K_{1}(1+2 c)},\left(\frac{\epsilon}{2}\right)^{q}\left(\frac{K_{1}}{c^{2}}+\frac{K_{1}}{c}+\frac{K_{1} C_{2}^{m}}{c\left[\frac{m}{p(m-1)}-1\right]}\right)^{-q}\right\},
$$

where

$$
\begin{aligned}
K_{1} & :=\max \{f(u): u \in[0, \gamma+\epsilon]\}, \\
\rho_{1} & :=\inf \left\{\frac{f(u)-K}{c}: \gamma+\frac{\epsilon}{2} \leq u \leq \gamma+\epsilon\right\}, \\
C_{2} & :=\left(p^{\frac{1}{p}} q^{\frac{1}{q}}\left(\frac{(m-1) \rho_{1}}{c}\right)^{1-\frac{1}{q}}\right)^{\frac{1}{1-m}},
\end{aligned}
$$

and $\delta_{0}$ is defined in the assertion (i). We claim that this $\delta$ satisfies the required property. Fix $a \in(\gamma-\delta, \gamma)$. Without loss of generality, we may assume that $b=b(a, c)>\gamma+\epsilon / 2$. Hence we can choose a $t_{2}>t_{1}$ with the property that $U\left(t_{2}\right)=\gamma+\epsilon / 2$.

Define $T_{2}:=\sup \left\{t \in\left(t_{2}, \infty\right): U(t)<\gamma+\epsilon\right\}$. Then it remains to show that $T_{2}=\infty$. In fact, by Young's inequality, we have

$$
\begin{aligned}
& V^{1-m}\left(t_{2}\right)+\frac{(m-1) \rho_{1}}{c}\left(t-t_{2}\right) \\
= & \frac{(m-1) \rho_{1}}{c}\left(\frac{\left[q^{\frac{1}{q}} V^{\frac{1-m}{q}}\left(t_{2}\right)\left(\frac{c}{(m-1) \rho_{1}}\right)^{\frac{1}{q}}\right]^{q}}{q}+\frac{\left[p^{\frac{1}{p}}\left(t-t_{2}\right)^{\frac{1}{p}}\right]^{p}}{p}\right) \\
\geq & C_{2}^{1-m} V^{\frac{1-m}{q}}\left(t_{2}\right)\left(t-t_{2}\right)^{\frac{1}{p}}
\end{aligned}
$$

for all $t \geq t_{2}$. It follows from the definition of $\rho_{1},(2.14)$ and the above estimate that

$$
\begin{aligned}
V(t) & =\left(\frac{1}{V^{1-m}\left(t_{2}\right)+(m-1) \int_{t_{2}}^{t}[(f(U(s))-K) / c] d s}\right)^{\frac{1}{m-1}} \\
& \leq\left(\frac{1}{V^{1-m}\left(t_{2}\right)+(m-1) \rho_{1}\left(t-t_{2}\right) / c}\right)^{\frac{1}{m-1}} \\
& \leq C_{2} V^{\frac{1}{q}}\left(t_{2}\right)\left(t-t_{2}\right)^{\frac{1}{p(1-m)}} \\
& \leq C_{2} V^{\frac{1}{q}}\left(t_{1}\right)\left(t-t_{2}\right)^{\frac{1}{p(1-m)}} \quad\left(\text { since } V\left(t_{1}\right)=\sup _{s \in \mathbb{R}} V(s)\right) \\
& \leq C_{2} \delta^{\frac{1}{q}}\left(t-t_{2}\right)^{\frac{1}{p(1-m)}} \quad(\text { by the assertion (i)) }
\end{aligned}
$$

for all $t \in\left(t_{2}, T_{2}\right)$. Now we use (2.10) to estimate $U^{\prime}\left(t_{2}\right)$ as follows:

$$
\begin{aligned}
U^{\prime}\left(t_{2}\right) & =\int_{-\infty}^{t_{2}} e^{-c\left(t_{2}-s\right)} f(U(s)) V^{m}(s) d s \\
& \leq K_{1} V^{m}\left(t_{1}\right) \int_{-\infty}^{t_{2}} e^{-c\left(t_{2}-s\right)} d s \\
& =\frac{K_{1} V^{m}\left(t_{1}\right)}{c}<\frac{K_{1} \delta}{c} .
\end{aligned}
$$


With these preparations, we can come to the assertion of the claim. First we show that $T_{2}-t_{2}>2$. Indeed, combining the above estimate on $U^{\prime}\left(t_{2}\right)$ with (2.8), this yields that for all $t \in\left(t_{2}, T_{2}\right)$ we have

$$
\begin{aligned}
U(t) & <U\left(t_{2}\right)+\frac{U^{\prime}\left(t_{2}\right)}{c}+\frac{K_{1}}{c} \int_{t_{2}}^{t} V^{m}(s) d s \\
& \leq U\left(t_{2}\right)+\frac{U^{\prime}\left(t_{2}\right)}{c}+\frac{K_{1} V^{m}\left(t_{1}\right)}{c}\left(t-t_{2}\right) \\
& <\gamma+\frac{\epsilon}{2}+\frac{K_{1} \delta}{c^{2}}+\frac{K_{1} \delta}{c}\left(t-t_{2}\right)
\end{aligned}
$$

This together with the definition of $\delta$ implies that $T_{2}-t_{2}>2$. (Otherwise, we have $U\left(T_{2}\right) \leq \gamma+3 \epsilon / 4$, a contradiction to the definition of $T_{2}$.) Therefore, by using (2.8) again, it follows that for all $t \in\left(t_{2}, T_{2}\right)$, we have

$$
\begin{aligned}
U(t) & <U\left(t_{2}\right)+\frac{U^{\prime}\left(t_{2}\right)}{c}+\frac{K_{1}}{c} \int_{t_{2}}^{t_{2}+1} V^{m}(s) d s+\frac{K_{1}}{c} \int_{t_{2}+1}^{t} V^{m}(s) d s \\
& \leq U\left(t_{2}\right)+\frac{U^{\prime}\left(t_{2}\right)}{c}+\frac{K_{1} V^{m}\left(t_{1}\right)}{c}+\frac{K_{1}}{c} \int_{t_{2}+1}^{t} V^{m}(s) d s \quad\left(\text { since } V\left(t_{1}\right)=\sup _{s \in \mathbb{R}} V(s)\right) \\
& <\gamma+\frac{\epsilon}{2}+\frac{K_{1} \delta}{c^{2}}+\frac{K_{1} \delta}{c}+\frac{K_{1}}{c} \int_{t_{2}+1}^{t} C_{2}^{m} \delta^{\frac{1}{q}}\left(s-t_{2}\right)^{\frac{m}{p(1-m)}} d s \\
& \left.<\gamma+\frac{\epsilon}{2}+\frac{K_{1} \delta^{\frac{1}{q}}}{c^{2}}+\frac{K_{1} \delta^{\frac{1}{q}}}{c}+\frac{K_{1} C_{2}^{m} \delta^{\frac{1}{q}}}{c\left[\frac{m}{p(m-1)}-1\right]} \quad(\text { by (3.1) }) \text { and } \delta \in(0,1)\right) \\
\leq &
\end{aligned}
$$

This implies that $T_{2}=\infty$ and hence the proof is completed.

The proof of the following lemma is only a slight modification of [1, Lemma 2.3], and so we omit it.

LEMMA 3.3. It follows that

$$
b(a, c)>a+K \int_{a}^{u_{0}} \frac{1}{f(u)} d u
$$

for any $a \in(0, \gamma)$ and $c>c(a)$, where $u_{0}$ is defined in the assumption (A2).

LEMma 3.4. For each given $a_{0} \in(0, \gamma)$ and $c$ such that $c>c(\hat{a})$ for all $\hat{a} \in\left(a_{0}-\delta_{0}, a_{0}+\delta_{1}\right)$ and for some $\delta_{0} \in\left(0, a_{0}\right)$ and $\delta_{1} \in\left(0, \gamma-a_{0}\right)$, the function $b(a, c)$ is continuous at $a=a_{0}$.

Proof. For each given $a \in\left(a_{0}-\delta_{0}, a_{0}+\delta_{1}\right)$, we let $\left(U(t ; a), U^{\prime}(t ; a), V(t ; a)\right)$ be the solution of the problem (1.4)-(1.5) such that $U(0 ; a)=\gamma$. We note that $\left(U(t ; a), U^{\prime}(t ; a)\right.$, $V(t ; a))$ with $a \in\left(a_{0}-\delta_{0}, a_{0}+\delta_{1}\right)$ is globally defined by the choice of $c$ and Lemma 2.4. 
Set

$$
\begin{aligned}
b_{0} & =b\left(a_{0}\right), \quad \epsilon_{0}=\frac{1}{4}\left(b_{0}-\gamma\right), \quad \rho_{0}=\inf \left\{\frac{f(u)-K}{c}: u \in\left[b_{0}-\epsilon_{0}, b_{0}+\epsilon_{0}\right]\right\}, \\
K_{0} & =\max \left\{f(u): u \in\left[b_{0}-\epsilon_{0}, b_{0}+\epsilon_{0}\right]\right\}, \quad C_{2}:=\left(p^{\frac{1}{p}} q^{\frac{1}{q}}\left(\frac{(m-1) \rho_{0}}{c}\right)^{1-\frac{1}{q}}\right)^{\frac{1}{1-m}} .
\end{aligned}
$$

Here we choose $p, q>1$ such that $m /[p(m-1)]>1$ and $1 / p+1 / q=1$. Fix an $\epsilon \in\left(0, \epsilon_{0} / 2\right)$. Using the fact that $\lim _{t \rightarrow+\infty}\left(U\left(t ; a_{0}\right), U^{\prime}\left(t ; a_{0}\right), V\left(t ; a_{0}\right)\right)=\left(b_{0}, 0,0\right)$, we can choose a sufficiently large $t_{2}$ such that

$$
\begin{aligned}
& b_{0}-\frac{1}{4} \epsilon<U\left(t ; a_{0}\right)<b_{0}, \quad 0<U^{\prime}\left(t ; a_{0}\right)<\frac{c}{32} \epsilon, \text { and } \\
& 0<V\left(t ; a_{0}\right)<\min \left\{\left(\frac{3 c}{64 K_{0}} \epsilon\right)^{\frac{1}{m}},\left(\frac{3 c}{64 K_{0}} \cdot \frac{\left[\frac{m}{p(m-1)}-1\right]}{C_{2}^{m}} \epsilon\right)^{\frac{q}{m}}\right\}
\end{aligned}
$$

for all $t \geq t_{2}$. Recall that for any negative $t$ with $|t| \gg 1,\left(U(t ; a), U^{\prime}(t ; a), V(t ; a)\right)$ lies on the centre manifold of the system (2.3) at $(a, 0,0)$. Hence by using the representation (2.22) of this centre manifold and the proof of existence of solutions to the system (2.17), we see that $\left(U(t ; a), U^{\prime}(t ; a), V(t ; a)\right)$ is continuous on $\left(-\infty, \hat{t}_{2}\right]$ for some negative $\hat{t}_{2}$ with $\left|\hat{t}_{2}\right| \gg 1$. Then, in the interval $\left[\hat{t}_{2}, t_{2}\right]$, we use the standard theory for the continuous dependence of solutions of differential equations on the parameters to conclude that there is a positive $\delta$ such that for all $a \in\left(a_{0}-\delta, a_{0}+\delta\right) \subset\left(a_{0}-\delta_{0}, a_{0}+\delta_{1}\right)$, the following hold:

$$
\begin{aligned}
& b_{0}-\frac{1}{2} \epsilon<U\left(t_{2} ; a\right)<b_{0}+\frac{1}{2} \epsilon, \quad 0<U^{\prime}\left(t_{2} ; a\right)<\frac{c}{16} \epsilon, \text { and } \\
& 0<V\left(t_{2} ; a\right)<\min \left\{\left(\frac{3 c}{32 K_{0}} \epsilon\right)^{\frac{1}{m}},\left(\frac{3 c}{32 K_{0}} \cdot \frac{\left[\frac{m}{p(m-1)}-1\right]}{C_{2}^{m}} \epsilon\right)^{\frac{q}{m}}\right\} .
\end{aligned}
$$

Here we have used the fact that $U^{\prime}(\cdot ; a)>0$ and $V(\cdot ; a)>0$ on $\mathbb{R}$.

Set $T_{2}:=\sup \left\{t \in\left(t_{2}, \infty\right):\left|U(t ; a)-b_{0}\right|<\epsilon\right\}$. Then it remains to show that $T_{2}=\infty$. First, by using a similar argument as in (3.1), we have that

$$
V(t ; a) \leq C_{2} V^{\frac{1}{q}}\left(t_{2}, a\right)\left(t-t_{2}\right)^{\frac{1}{p(1-m)}}
$$

for all $t \in\left(t_{2}, T_{2}\right)$. Next we show that $T_{2}-t_{2}>2$. Indeed, by applying (2.8) (with $\left.t_{0}=t_{2}\right)$ and using the definition of $K_{0}$, it follows that

$$
\begin{aligned}
U(t ; a) & <U\left(t_{2} ; a\right)+\frac{U^{\prime}\left(t_{2} ; a\right)}{c}+\frac{K_{0}}{c} \int_{t_{2}}^{t} V^{m}(s ; a) d s \\
& \leq U\left(t_{2} ; a\right)+\frac{U^{\prime}\left(t_{2} ; a\right)}{c}+\frac{K_{0} V^{m}\left(t_{2} ; a\right)}{c}\left(t-t_{2}\right) \quad\left(\text { since } V^{\prime}(\cdot ; a)<0 \text { on }\left[t_{2}, T_{2}\right)\right) \\
& \leq b_{0}+\frac{1}{2} \epsilon+\frac{1}{16} \epsilon+\frac{3 \epsilon}{32}\left(t-t_{2}\right) \quad(\text { by }(3.2))
\end{aligned}
$$

for all $t \in\left(t_{2}, T_{2}\right)$. This implies that $T_{2}-t_{2}>2$, since otherwise, we have $U\left(T_{2} ; a\right) \leq$ $b_{0}+3 \epsilon / 4$, a contradiction to the definition of $T_{2}$. Therefore, with the aid of (2.8), the 
fact that $V^{\prime}(\cdot ; a)<0$ on $\left[t_{2}, T_{2}\right)$ and the definition of $K_{0}$, it follows that

$$
\begin{aligned}
U(t ; a) & <U\left(t_{2} ; a\right)+\frac{U^{\prime}\left(t_{2} ; a\right)}{c}+\frac{K_{0}}{c} \int_{t_{2}}^{t_{2}+1} V^{m}(s ; a) d s+\frac{K_{0}}{c} \int_{t_{2}+1}^{t} V^{m}(s ; a) d s \\
& \leq U\left(t_{2} ; a\right)+\frac{U^{\prime}\left(t_{2} ; a\right)}{c}+\frac{K_{0} V^{m}\left(t_{2} ; a\right)}{c}+\frac{K_{0}}{c} \int_{t_{2}+1}^{t} V^{m}(s ; a) d s \\
& \leq b_{0}+\frac{1}{2} \epsilon+\frac{1}{16} \epsilon+\frac{3}{32} \epsilon+\frac{K_{0}}{c} \int_{t_{2}+1}^{t} C_{2}^{m} V^{\frac{m}{q}}\left(t_{2} ; a\right)\left(s-t_{2}\right)^{\frac{m}{p(1-m)}} d s
\end{aligned}
$$

$$
\leq \quad b_{0}+\frac{21}{32} \epsilon+\frac{K_{0} C_{2}^{m} V^{\frac{m}{q}}\left(t_{2} ; a\right)}{c\left[\frac{m}{p(m-1)}-1\right]} \leq b_{0}+\frac{3}{4} \epsilon \quad(\text { by }(\underline{3.2}))
$$

for all $t \in\left(t_{2}, T_{2}\right)$. This implies that $T_{2}=\infty$, thereby completing the proof.

3.2. Proofs of Theorems 1.1 1.3. Now we are ready to prove Theorems 1.11 .3 . Indeed, the first part of Theorem 1.1 and Theorem 1.2 immediately follow from Lemmas 2.4. 2.2 and 3.1 The first part of Theorem 1.3 follows from Lemmas 3.2, 3.3 and 3.4

Next, we will show that for each given $b_{0}>\gamma$, there is a nonnegative constant $\bar{c}\left(b_{0}\right)$ such that for each $c>\bar{c}\left(b_{0}\right)$, there exists a solution $(U, V)$ to (1.4)-(1.5) for some $a \in$ $(0, \gamma)$. Indeed, by Lemmas 3.3 and the fact that

$$
\int_{0^{+}}^{u_{0}} \frac{1}{f(u)} d u=+\infty
$$

we can choose an $\hat{a} \in(0, \gamma)$ such that $b(\hat{a}, c)>b_{0}$ for all $c>c(\hat{a})$. Set $\bar{c}\left(b_{0}\right):=$ $\sup _{a \in[\hat{a}, \gamma)} c(a)$. Note that $\bar{c}\left(b_{0}\right)=0$ for $m \in(1,2]$ by Lemma 2.4. Recall from part (ii) of Lemma 3.2 that $\lim _{a \rightarrow \gamma^{-}} b(a, c)=\gamma$ for each $c>0$. Together with Lemma 3.4. we can conclude that for each $c>\bar{c}\left(b_{0}\right)$ there exists an $a\left(b_{0}, c\right) \in(\hat{a}, \gamma)$ such that $b\left(a\left(b_{0}, c\right), c\right)=b_{0}$. Hence the "converse" part of Theorem 1.1 follows.

Finally, we turn to the proof of the second part of Theorem 1.3. Given $0<a_{1}<a_{2}<$ $\gamma$, set $b_{i}:=\lim _{t \rightarrow+\infty} U\left(t ; a_{i}\right), i=1,2$. Let $\left(U\left(t ; a_{i}\right), U^{\prime}\left(t ; a_{i}\right), V\left(t ; a_{i}\right)\right), i=1,2$, be the solution of the problem (1.4) -(1.5) such that $U\left(0 ; a_{i}\right)=\gamma$. We can conclude from part (v) of Lemma 2.1 and part (iv) of Lemma 2.2 that $U\left(t ; a_{1}\right)<U\left(t ; a_{2}\right), U^{\prime}\left(t ; a_{1}\right)<U^{\prime}\left(t ; a_{2}\right)$, and $V\left(t ; a_{1}\right)<V\left(t ; a_{2}\right)$ for negative $t$ with $|t| \gg 1$. Then following the arguments of 1 , pp. 671-673] we have $b_{1}>b_{2}$.

Acknowledgements. The authors would like to thank Professor Masayasu Mimura for bringing our attention to this problem. The authors would like to thank the referees for helpful comments on this paper.

\section{REFERENCES}

[1] S. Ai and W. Huang, Traveling waves for a reaction-diffusion system in population dynamics and epidemiology, Proc. Roy. Soc. Edinburgh Sect. A 135A (2005), 663-675. MR 2173333(2006e:35188)

[2] S. Ai and W. Huang, Traveling wavefronts in combustion and chemical reaction models, Proc. Roy. Soc. Edinburgh Sect. A 137A (2007), 671-700. MR2345776 (2008g:35106)

[3] R. Aris, P. Gray and S. K. Scott, Modeling of cubic autocatalysis by successive bimolecular steps, Chem. Eng. Sci. 43 (1988), 207-211. 
[4] J. Billingham and D.J. Needham, The development of travelling waves in quadratic and cubic autocatalysis with unequal diffusion rates. I. Permanent form travelling waves, Philos. Trans. Roy. Soc. Ser. A 334 (1991), 1-24. MR 1155096 (92m:80020)

[5] J. Billingham and D.J. Needham, The development of travelling waves in quadratic and cubic autocatalysis with unequal diffusion rates. II. An initial value problem with an immobilized or nearly immobilized autocatalyst, Philos. Trans. Roy. Soc. Ser. A 336 (1991), 497-539. MR1133118 (93a:80012)

[6] N.T.J. Bailey, The mathematical theory of infectious diseases, Griddin, London, 1975.

[7] M. Ballyk, L. Dung, D. A. Jones, and H. L. Smith, Effects of random motility on microbial growth and competition in a flow reactor, SIAM J. Appl. Math. 59 (1999), 573-596. MR1654407 (2001a:92027)

[8] J. Carr, Applications of centre manifold theory, Springer-Verlag, New York, 1981. MR635782 (83g:34039)

[9] X. Chen and Y. Qi, Sharp estimates on minimum traveling wave speed of reaction diffusion systems modelling autocatalysis, SIAM J. Math. Anal. 39 (2007), 437-448. MR2338414 (2008h:34083)

[10] S. Dunbar, Traveling wave solutions of diffusive Lotka-Volterra equations, J. Math. Biol. 17 (1983), 11-32. MR707221 (84j:92031)

[11] P. Gray, Instabilities and oscillations in chemical reactions in closed and open systems, Proc. Roy. Soc. London Ser. A 415 (1988), 1-34.

[12] Y. Hosono and B. Ilyas, Existence of traveling waves with any positive speed for a diffusive epidemic model, Nonlin. World 1 (1994), 277-290. MR1303097 (95k:92021)

[13] Y. Hosono and B. Ilyas, Travelling waves for a simple diffusive epidemic model, Math. Models Meth. Appl. Sci. 5 (1995), 935-966. MR.1359215 (96j:35248)

[14] Y. Hosono, Phase plane analysis of travelling waves for higher order autocatalytic reaction-diffusion systems, Discrete Contin. Dyn. Syst. Ser. B 8 (2007), 115-125. MR 2300326 (2008c:35148)

[15] W. Huang, Travelling waves for a biological reaction-diffusion model, J. Dynam. Diff. Eqns. 16 (2004), 745-765. MR2109164 (2005g:35175)

[16] A. Källén, Thresholds and travelling waves in an epidemic model for rabies, Nonlinear Anal. TMA 8 (1984), 851-856. MR753763 (86h:92042)

[17] W. O. Kermack and A. G. McKendric, Contribution to the mathematical theory of epidemics, Proc. Roy. Soc. A 115 (1927), 700-721.

[18] C. R. Kennedy and R. Aris, Travelling waves in a simple population model involving growth and death, Bull. Math. Biol. 42 (1980), 397-429. MR661329 (84f:92041)

[19] J. H. Merkin and D. J. Needham, The development of travelling waves in a simple isothermal chemical system. II. Cubic autocatalysis with quadratic and linear decay, Proc. Roy. Soc. London Ser. A 430 (1990), 315-345. MR1068302 (91i:80008)

[20] J. H. Merkin and D. J. Needham, The development of travelling waves in a simple isothermal chemical system. IV. Quadratic autocatalysis with quadratic decay, Proc. Roy. Soc. London Ser. A 434 (1991), 531-554.

[21] J. H. Merkin and D. J. Needham, The development of travelling waves in a simple isothermal chemical system with general orders of autocatalysis and decay, Philos. Trans. Roy. Soc. London Ser. A 337 (1991), 261-274. MR1143726 (93a:80013)

[22] J. D. Murray, Mathematical biology. I: An introduction, Springer-Verlag, New York, 2004. MR 1908418 (2004b:92003)

[23] Y. Qi, The development of travelling waves in cubic auto-catalysis with different rates of diffusion, Physica D. 226 (2007), 129-135. MR2296235 (2007k:35270)

[24] A. Saul and K. Showalter, Propagating reaction-diffusion fronts, in: R.J. Field and M. Burger (Eds.), Oscillations and Traveling waves in chemical systems, Wiley, New York, 1984.

[25] H. L. Smith and X. Q. Zhao, Travelling waves in a bio-reactor model, Nonlinear Anal. Real World Appl. 5 (2004), 895-909. MR2085700 (2005g:35161)

[26] V. G. Voronkov and N. N. Semenov, Zh. Fiz. Khim. 13 (1939), 1695.

[27] J. Xin, Front propagation in heterogeneous media, SIAM Rev. 42 (2000), 161-230. MR.1778352 (2001i:35184)

[28] A. N. Zaikin and A. M. Zhabotinskii, Concentration wave propagation in two-dimensional liquidphase self-oscillating system, Nature 225 (1970), 535-537. 